\title{
LEOPARDI POLITICO TRA AMOR PATRIO E FRATELLANZA UNIVERSALE
}

di Giulio Di Donato

Mai come durante le settimane di dolore e preoccupazione legate all'emergenza Coronavirus si sono manifestati così tanti atteggiamenti di fierezza nazionale: un sussulto di orgoglio generato da un concorso drammatico di circostanze che è diventato quasi un moto spontaneo e liberatorio utile ad esorcizzare le paure e le tensioni accumulate.

Il senso di comunità inevitabilmente si rafforza soprattutto nei momenti di smarrimento collettivo, quando si cerca un 'noi' che ci protegga e rassicuri. Va compresa dunque questa forma di patriottismo che nasce dall'angoscia e dal desiderio di non sentirsi soli in frangenti tanto difficili.

Certo, tutto questo non può valere in eterno: dopo una prima fase di union sacrée, se una situazione di grave crisi si protrae a lungo nel tempo e si ha l'impressione di non essere veramente protetti, il tessuto sociale si sfalda e si precipita nella caccia al nemico interno.

Inoltre, proprio durante il periodo di isolamento forzato ci siamo resi conto di come la dimensione del 'fuori' sia necessaria per guardare avanti e costruire progetti. Per vivere quel presente ristretto dovevamo difatti supporre un 'fuori', inteso sia come il mondo esterno a cui eravamo abituati, sia come 'dopo', come momento in cui potevamo riprendere pienamente la vita di sempre. Il 'fuori' e il 'dopo' sono i punti d'appoggio per agire 'qui' ed 'ora'. Ma erano in buona parte finzioni. Il 'fuori' esisteva solo parzialmente, mentre il 'dopo' restava avvolto da un alone di imponderabilità, su cui potevamo solo fare ipotesi molto generali. E però l'elemento più interessante è che in quel

Dipartimento di Scienze giuridiche, Università di Salerno. 
tempo sospeso abbiamo avuto un bisogno vitale di immaginare questo 'mondo di fuori', un'ipotesi immaginaria ma assolutamente necessaria.

Molto probabilmente quella esperienza prolungata di confinamento domestico obbligatorio ci abituerà ad una sorta di restrizione delle relazioni sociali e degli spazi d'azione destinata a incidere a lungo sui nostri comportamenti futuri, spingendoci a preferire sempre più $i$ rapporti di vicinato, la prossimità, la frequentazione di cerchie ristrette e dunque, in un certo senso, la stanzialità. $\mathrm{Ci}$ aspetta dunque un ritorno al localismo, riscoperto sempre più alla stregua di una dimensione virtuosa in ogni ambito, compreso quella politica (da qui la possibile spinta a ripensare l'autonomismo sul piano politico-istituzionale, ma ancora prima sul piano culturale e del sentimento collettivo).

Quel che possiamo fare, intanto, è provare a trarre qualche lezione dalla gestione della crisi emergenziale. La prima ci ricorda l'importanza del nostro servizio sanitario pubblico che, nonostante anni di tagli e spending review, rappresenta un'eccellenza della quale essere orgogliosi. Una seconda lezione ci impone di riconoscere la centralità dell'intervento pubblico-statuale e il peso della dimensione nazionale, la quale garantisce quel minimum di identificazione e di missione comune, quindi di coesione e di lealtà politica, quanto mai necessario in un momento in cui c'è da affidarsi al senso di sacrificio e di responsabilità collettiva.

Ormai acquisisce sempre più evidenza il fatto che solo uno Stato nazionale è in grado di garantire un rapporto equilibrato - per lo più democratico - fra la dimensione geopolitica e il sentimento di appartenenza e di "intimità" fra i cittadini e già per questo svolge una funzione difficilmente sostituibile, anche nei confronti degli eccessi secessionistici delle rivendicazioni etniche ${ }^{1}$.

Queste indicazioni potrebbero, inoltre, aiutarci a impostare nel modo giusto il rapporto fra la dimensione nazionale e quella sovranazionale, rafforzando e confermando convincimenti maturati ben prima dell'emergenza sanitaria.

Crediamo infatti che la battaglia politica prioritaria dei nostri giorni sia quella di ridefinire il nesso nazionale/sovranazionale (con particolare riferimento alla posizione del nostro paese in ambito UE)

1 D. Zolo, Globalizzazione, Roma-Bari, Laterza, 2006, p. 79. 
secondo una prospettiva alternativa a quella egemone negli ultimi quarant'anni. Le sfide poste dai processi di (dis)integrazione globale degli ultimi quattro decenni sollecitano ormai da tempo tessiture nuove tra politica, diritto ed economia, che siano in grado di risolvere positivamente la contraddizione - già individuata da Gramsci all'inizio degli anni Trenta - tra il cosiddetto nazionalismo della politica e il cosmopolitismo dell'economia.

I fatti degli ultimi mesi avvalorano la tesi di chi crede che la soluzione non può che passare per un rinnovato protagonismo degli Stati nazionali (nella loro variante democratico-costituzionale) e per un contemporaneo rilancio della cooperazione internazionale; due momenti che appaiono - se opportunamente declinati - come stretti da un nesso di interdipendenza funzionale, dove il primo sollecita il secondo, e questo potenzia e rafforza l'altro. Il modello a cui guardare ci indirizza verso una forma di internazionalismo "equilibrato" che restituisca agli Stati la capacità di governare e orientare i processi economici², pur nella consapevolezza - mai così evidente come in questo periodo - che le democrazie nazionali devono confrontarsi con problemi che sfuggono in parte alla loro capacità di controllo e gestione (dal governo dei processi migratori ai flussi della comunicazione globale, dai problemi di approvvigionamento energetico agli effetti prodotti dalla guerra e dal terrorismo e via discorrendo) e che è altamente improbabile pensare di affrontare in un'ottica puramente

2 Nel cosiddetto trentennio glorioso (1945-1975) si è riusciti a instaurare e a salvaguardare, qui in Europa, un compromesso avanzato fra capitale e lavoro finché l'economia è rimasta in gran parte incapsulata dentro il contesto degli Stati nazionali. Nel corso degli ultimi decenni c'è stata un'indubbia sottovalutazione di gran parte delle forze di sinistra dei rischi derivanti dai processi di internazionalizzazione del capitalismo maturo e dai nuovi assetti politico-istituzionali che si andavano creando a livello sovranazionale, e ci si è ritrovati, senza troppo accorgersene o preoccuparsene, ad agire dentro un terreno di gioco che si è fatto col tempo assai ostile e inospitale. Tutti gli strumenti di cui si era dotato il movimento operaio durante il "secolo breve" per governare e orientare i processi economici, dagli anni ' 70 ad oggi, sono stati a poco a poco spuntati. Prima la globalizzazione neoliberista, poi il cosiddetto pilota automatico messo in moto dalla tecnocrazia europea hanno svuotato le istituzioni democratiche nazionali di poteri e funzioni e immobilizzato la politica dentro un recinto. Il movimento operaio è stato protagonista del secolo scorso, non solo perché difendeva gli ultimi, ma perché aveva creato degli strumenti di lotta straordinari. Con la globalizzazione e la nuova architettura europea sono venuti meno i vecchi strumenti dell'agire politico della sinistra. 
domestica una grave minaccia al destino dell'umanità come quella rappresentata dalle nuove pandemie.

In questo, come in altri casi, anche se il punto di partenza si situa sul terreno nazionale, la risposta non può che coinvolgere l'intera comunità internazionale, perché nessun paese al mondo può pensare di salvarsi da solo.

\section{La società mezzana}

Guardando alla storia dell'umanità, le epidemie rappresentano una tragica costante; ce ne sono poi alcune - come nel caso del Coronavirus - che coinvolgono il pianeta intero. In un passato non troppo lontano abbiamo avuto la spagnola e, ancora fresche nella memoria dei nostri contemporanei, ci sono l'AIDS e l'asiatica.

Fu negli anni del colera a Napoli che Leopardi scrisse, pensando allo "sterminator Vesevo" e alla sorte di Pompei ed Ercolano, quel capolavoro di poesia e filosofia che è La ginestra, che soccomberà al fuoco del vulcano, "ma più saggia, ma tanto/meno inferma dell'uom" che si credeva e si crede onnipotente, in grado di dominare e addomesticare la natura nel modo a lui più utile e congeniale.

Oggi che ci confrontiamo con gli effetti di una nuova pandemia può essere utile ripercorrere l'itinerario leopardiano, che proprio con $L a$ ginestra "salta" dall'amor patrio alla fratellanza universale, senza stabilire o presupporre una contraddizione fra i due momenti.

Come è noto Leopardi, nello Zibaldone, magnifica l'idea della "società mezzana" o di "mezzana grandezza" che, a ben vedere, altro non è che "una nazione" ("la mezzana civiltà trionferà in tutto il mondo").

"La patria moderna - scrive il grande poeta recanatese - dev'essere abbastanza grande, ma non tanto che la comunione d'interessi non vi si possa trovare, come chi ci volesse dare per patria l'Europa. La propria nazione, coi suoi confini segnati dalla natura, è la società che ci conviene. E conchiudo che senza amor nazionale non si dà virtù grande"3.

3 G. LeOPardi, Pensieri di varia filosofia e bella letteratura (Zibaldone), Firenze, Successori Le Monnier, 1898, volume secondo, p. 248. 
Sempre Leopardi in un altro celebre passo:

"Quando tutto il mondo fu cittadino Romano, Roma non ebbe più cittadini; e quando cittadino Romano fu lo stesso che cosmopolita, non si amò né Roma né il mondo: l'amor patrio di Roma divenuto cosmopolita, divenne indifferente, inattivo e nullo: e quando Roma fu lo stesso che il mondo, non fu più patria di nessuno, e i cittadini romani, avendo per patria il mondo, non ebbero nessuna patria, e lo mostrarono col fatto"4.

La conclusione da trarre, agli occhi di Leopardi, è la seguente: in assenza di un comune interesse e di un legame patriottico (così come senza "illusioni"), una comunità politica non può prosperare, né possono essere temperati e ridimensionati gli egoismi dei suoi cittadini. Il modello è quello delle "antiche e poche ristrette società" nelle quali "l'amor proprio fu ridotto ad ... amor di corpo o di patria"s.

Se viene meno l'amor di patria l'agire dei singoli perde i suoi criteri di orientamento. Non si allarga certo l'orizzonte di riferimento, l'uomo non diventa improvvisamente cittadino del mondo, ma accade piuttosto il contrario: il singolo fa di sé stesso una repubblica in perpetuo conflitto con le altre. Altro che abolizione delle ostilità: la scomparsa dell'amor di patria ne promuove invece lo slittamento sul piano individuale. L'amor di preferenza, se non si radica nell'idea di nazione, quale luogo privilegiato d'espressione, favorendo una sintesi positiva fra gli interessi dei singoli e quelli della collettività, non può che rifluire sull'individuo, dando luogo ad un vero e proprio sistema dell'egoismo.

"La fola dell' amore universale, del bene universale, col qual bene ed interesse, non può mai congiungersi il bene e l'interesse dell'individuo, che travagliando per tutti non travaglierebbe per sé, né per superar nessuno, come la natura vuol ch'ei travagli, ha prodotto l'egoismo universale. Non si odia più lo straniero? ma si odia il compagno, il concittadino, l'amico, il padre, il figlio; ma l'amore è sparito affatto dal mondo, sparita la fede, la giustizia, l'amicizia, l'eroismo, ogni virtù, fuorché l'amor di sé stesso. Non si hanno più nemici nazionali? ma si hanno nemici privati, e tanti quanti son gli uomini; ma non si hanno più amici di sorta alcuna, né doveri se non verso sé stesso"6.

4 G. LeOPardi, Pensieri di varia filosofia e bella letteratura (Zibaldone), cit., p. 2.

5 G. LeOPARDI, Pensieri di varia filosofia e bella letteratura (Zibaldone), cit., p. 235.

6 G. Leopard, Pensieri di varia filosofia e bella letteratura (Zibaldone), cit., p. 244. 
Insomma, quando l'inimicizia non si esercita più sul confine fra noi e loro, si frantuma e si diffonde lungo un asse che contrappone l'io a tutti gli altri.

Ed è un'illusione pensare che l'abbandono dell'amor patrio possa condurre ad una più larga solidarietà: dietro la melassa dell'universale solidarietà, si nasconde difatti il dominio universale dell'egoismo privato e si afferma "la teoria del non fare bene a nessuno"

\section{La social catena}

Forzando un po' il discorso, sembra quasi che in Leopardi ci sia la consapevolezza per cui ogni comunità politica non può esistere senza una dialettica dentro/fuori. Il mondo - come sosterrà un secolo dopo Carl Schmitt - è un pluriverso e non un universo, a causa della tendenza degli esseri umani a riunirsi in comunità determinate $\mathrm{e}$ irriducibili le une alle altre (che non significa per nulla chiuse o in opposizione fra loro). Da qui il topos del "nemico" - questo sì ben presente in Leopardi - da non intendere come appello ad un sentimento di odio dei confronti dello straniero, ma in ragione della necessità della sua esistenza, come fattore di unità e coesione interna.

Proprio nella Ginestra l'affratellamento degli uomini appare possibile solo perché sullo sfondo si staglia un nuovo nemico: non più un nemico "umano e relativo (nemico ad alcuni e non ad altri), ma un nemico impersonale e assoluto (nemico di tutto il genere umano)"8. Questo nemico è la natura, contro la quale gli uomini affratellati sono chiamati a una "guerra comune". E proprio perché essi agiscono "contro l'empia natura" si possono stringere "in social catena". La natura è una nemica spesso crudele, ma è una nemica utile così come lo erano quei nemici esterni, la mobilitazione contro i quali permetteva di suscitare le virtù civili. La fratellanza di cui parla La ginestra non sembra comunque "affatto quella astratta degli ideali, delle fole, del progressismo cosmopolitico ... non sembra il frutto di un'aspirazione etica, ma appare come una fratellanza concreta, come una solidarietà

7 G. LeOPARDI, Pensieri di varia filosofia e bella letteratura (Zibaldone), cit., p. 240.

8 M. LuCiani, Lo sguardo profondo, Modena, Mucchi, 2017, pp. 116-117. 
combattiva e disincantata, frutto di una spinta materiale proveniente dalla comune soggezione di tutti gli uomini alle violenze e alle minacce della natura"9.

Da un lato abbiamo quindi la realtà delle "poche e ristrette società", nella quale l'individuo aderisce alla comunità senza dare pieno corso alla propria soggettività (la "bella vita etica" della polis greca per dirla con Hegel); dall'altro il sistema dell'egoismo moderno, che ama parlare di amore universale proprio perché sa che è vano ed impotente: ogni via di uscita sembra preclusa. Eppure c'è la possibilità di fare un "salto": può accadere, infatti, soprattutto in una situazione estrema che coinvolge tutti gli esseri umani, di alzare lo sguardo fino ad abbracciare l'umanità intera, sollevandosi ad una distanza infinita dalla quale tutto appare nella sua nudità e insignificanza. Dal punto di vista dell'infinito (sub specie aeternitatis direbbe Spinoza), l'esistenza dell'uomo ha ben poco significato e tutte le passioni, i clamori, gli affanni e i dolori che ci assediano ogni giorno non hanno alcun rilievo. Da quella distanza l'esistenza si scopre vicino al nulla, ma gli uomini appaiono tutti degni di compassione. Da quelle altezze la cerchia degli uomini appare ristretta, proprio come nelle repubbliche antiche, e così l'amor di preferenza può essere messo al servizio dell'utilità di tutti. Quel che è certo è che tra la natura e l'uomo c'è una distanza incolmabile, che nasce dall'irrilevanza per la prima della sorte del secondo, perché la natura è totalmente indifferente al destino dell'uomo e alle dinamiche della storia. Ma la distanza infinita della natura, la sua indifferenza e supremazia possono diventare una carta da giocare contro la natura stessa, una carta che impone agli uomini di stringersi in una guerra comune contro le sue offese e minacce ${ }^{10}$.

Evocare questo senso di sproporzione tra la nostra fragile finitezza e quegli interminabili spazi e quei sovrumani silenzi può consentirci quindi di rovesciare la nostra fragilità in spirito di fratellanza. Trasformando il sentimento della nostra vulnerabilità (pienamente smascherato dalla nuova emergenza sanitaria) in una battaglia comune in nome della solidarietà universale, non più vagamente sentimentale, ma concreta ed effettiva.

9 M. LuCiani, Lo sguardo profondo, cit., pp. 116-117.

${ }^{10}$ F. CAssano, Oltre il nulla, Roma-Bari, Laterza, 2003, p. 71 sgg.. 
Si tratterà allora di conservare la memoria delle emozioni che abbiamo vissuto in questi mesi difficili. La memoria delle emozioni, sì, perché la consapevolezza da sola non basta: come ci insegna Spinoza, la ragione deve farsi emozione per avere un qualche potere sulle passioni negative ${ }^{11}$. In questo caso l'appello ad un certo tipo di emozioni ha un significato ben preciso: preservare l'amor patrio dal rischio della degenerazione nazionalistica e impedire al principio di fratellanza universale di ridursi a vuota e falsa retorica.

\section{Cosa ci fa stare assieme?}

Questo breve richiamo al Leopardi politico ci consente di confrontarci con quello che è il punto forse più trascurato nell'ambito della cultura democratico-progressista, ovvero la necessità di legami prepolitici e di comunità per il buon funzionamento di una democrazia.

Come ha sostenuto Dahrendorf ${ }^{12}$, il sentimento di coesione e di lealtà politica fra i cittadini è una variabile essenziale di un sistema democratico. "E la coesione e la lealtà politica - per quanto astrattamente garantite nelle società moderne dallo strumento del diritto - suppongono comunque l'esistenza di legami prepolitici fra i membri del gruppo, rinviano ad una identità collettiva", come ha affermato Danilo Zolo in un suo prezioso e imprescindibile libro di diversi anni fa ${ }^{13}$. Ed è provato - continua Zolo - che la tenuta dei legami identitari si fa sempre più

11 Un affetto negativo "non può essere ostacolato né tolto se non da un affetto contrario e più forte dell'affetto da ostacolare", Etica, IV, Proposizione VII.

12 Si veda R. DAHRENDORF, Quadrare il cerchio, Roma-Bari, Laterza, 1995.

${ }^{13} \mathrm{D}$. Zolo, I signori della pace. Una critica del globalismo giuridico, Roma, Carocci, 2001. Di parere diverso Luigi Ferrajoli, che ha più volte ricordato l'interazione complessa, sperimentata anche a livello di formazione storica dello Stato moderno, fra senso comune di appartenenza e istituzioni giuridiche, tra unificazione politica e affermazione del principio di uguaglianza. "Se è vero - scrive l'illustre giurista - che coesione, legami prepolitici e identità collettiva della comunità internazionale formano i presupposti del progetto di una democrazia internazionale, è ancora più vero il contrario: è sull'uguaglianza dei diritti, quale garanzia di tutte le differenze d'identità personale, che si fonda la percezione degli altri come uguali e come consociati; ed è sulla garanzia dei propri diritti fondamentali come diritti uguali che matura il senso di appartenenza e identità collettiva di una comunità politica". (L. FerRajoli, Diritti fondamentali. Un dibattito teorico, Roma-Bari, Laterza, 2015, si legga l'intero paragrafo 5 del capitolo 1) 
incerta via via che l'ambito geopolitico di uno Stato si dilata fino a includere culture molto diverse fra loro ${ }^{14}$. Neppure il più astratto "patriottismo dei diritti" può fare a meno, per così dire, di una qualche "intimità" fra i membri del gruppo (Böckenförde, grande giurista tedesco di fede socialdemocratica, utilizza a tal proposito il concetto di "omogeneità relativa"15): essi non possono essere dei soggetti "estranei" gli uni agli altri, l'estraneità è l'opposto della solidarietà democratica' ${ }^{16}$.

${ }^{14}$ R. DAHL (Sulla democrazia, Roma-Bari, Laterza, 2002) pone lo scarso pluralismo subculturale fra le condizioni favorevoli alla democrazia.

15 E. Bockenforde, Diritto e secolarizzazione. Dallo stato moderno all'Europa unita, Roma-Bari, Laterza, 2007. La sfera pubblica che Böckenförde immagina implica l'accordo di fondo su alcune idee-base del paradigma liberale, perché "uno Stato non può staccarsi completamente dalle proprie radici ed elevarsi per così dire a puro Stato razionale, che privo di cultura e di radici propri, tratta allo stesso modo tutte le tradizioni rilevanti per ethos e diritto, e pone sullo stesso piano tutte le manifestazioni pubbliche delle religioni. Ciò che negli ultimi anni è stato definito in modo inadeguato con 'cultura guida' possiede un proprio fondamento". Il testo è tratto da una lettera di Böckenförde inviata al cardinale Ratzinger il 30 aprile 2004, riportato in A. CAVALIERE, Le ragioni della secolarizzazione. Böckenförde tra diritto e teologia politica, Torino, Giappichelli, 2016, p. 106. In ogni società, sembra dirci l'autorevole giurista tedesco, c'è una cultura dominante, cioè quella che determina il quadro delle regole generali. Regole che non sono mai neutre, ma rappresentano e tutelano sempre determinati modelli di vita, determinati valori, frutto di una determinata storia e di specifiche tradizioni. Di conseguenza la cosiddetta multiculturalità non può sussistere senza costanti in comune, senza punti di orientamento $\mathrm{a}$ partire dai valori propri.

${ }^{16}$ Ben diverso è il punto di vista di Habermas, che parla a tal proposito di "solidarietà tra estranei". Scrive Habermas: "Io vedo 'l'esprit' del repubblicanesimo nel fatto che le forme e le procedure dello Stato costituzionale forgiano, con la loro modalità democratica di legittimazione, un nuovo livello di coesione sociale. La cittadinanza democratica fonda una solidarietà tra estranei relativamente astratta e comunque giuridicamente mediata. Si tratta di una forma d'integrazione realizzatasi come contesto di comunicazione coinvolgente in sé la socializzazione politica. [...] L'identità collettiva non è mai indipendente dal processo democratico, né può esistere 'prima' di questo stesso processo. Quel che lega assieme una 'nazione di cittadini' [..., è] il contesto intersoggettivamente condiviso di un'intesa possibile". Si veda J. HABERMAS, Una costituzione per l'Europa?, in G. ZAGREBELSKY, P. P. PorTinARO, J. LuTHER (a cura ), "Il futuro della costituzione", Torino, Einaudi, 1996, p. 373. "Le nazioni”, continua Habermas, "[possono] nascere solo dopo essersi lasciate alle spalle il difficile percorso che dalla comunanza etnica di compagni personalmente conosciuti conduce alla solidarietà giuridica di cittadini reciprocamente estranei". (J. HABERMAS, Una costituzione per l'Europa, cit.,) Altrove, guardando al processo di integrazione europea, ritorna su questi temi: "Il deficit democratico non si riferisce soltanto a quei regolamenti tra governi che, poggiando su accordi meramente pragmatici di attori collettivi, non possono evidentemente avere la forza legittimante di una società di cittadini politicamente costituita. Quel problema si riferisce anche alle influenze prodotte dalla glo- 
Insomma, l'identificazione con una qualche comunità concreta di appartenenza costituisce una risorsa necessaria al buon funzionamento di una democrazia. Se "manca un sostrato oggettivo, se manca il radicamento in una forma di vita le comunità politiche non hanno alcuna possibilità di costituirsi e di rimanere vitali" "17.

Diversamente dall'universalismo liberale, l'universalismo democratico è radicato in, e si riferisce a, uno specifico demos costituito da persone che sono unite da qualcosa di più dell'umanità e della ragione. La solidarietà civica è radicata nelle identità collettive particolari (che non hanno certo un fondamento essenzialista o assoluto, perché appartengono alla dimensione storico-culturale che come tale ha natura transeunte e relativa ${ }^{18}$ ); la solidarietà cosmopolitica si sostiene unicamente in virtù dell'universalismo morale dei diritti umani. Questo implica il riconoscimento che la cittadinanza democratica è una politica di identità che non può essere così astratta e senza fondamenti come lo è l'appartenenza all'umanità. D'altro canto, tuttavia, la cittadinanza democratica - come ha ben spiegato Nadia Urbinati ${ }^{19}$ - è una identità politica che educa i cittadini a vedere gli altri come uguali, e questo è ciò che rende la politica democratica un giuoco aperto all'auto-revisione e, soprattutto, le questioni relative ai confini dello Stato questioni sempre soggette a modifiche e ad aggiustamenti, nel tentativo di rendere il governo dei flussi migratori il più possibile conforme ai principi di umanità e giustizia e le politiche di integrazione degli immigrati il più possibile coerenti con i principi democratico-costituzionali ${ }^{20}$.

balizzazione sul sostrato culturale di solidarietà civica che si era già formato nel quadro dello Stato nazionale": J. HABERMAS, La costellazione postnazionale, Milano Feltrinelli, 1999, p. 46.

${ }^{17}$ M. LuCIani, Articolo 12, Roma, Carocci, 2018, pag. 75.

${ }^{18}$ Non si tratta quindi di assumere come naturali le configurazioni comunitarie e le identità collettive esistenti, rinunciando ad interrogarsi sui processi materiali della loro e produzione e riproduzione, nonché sui rapporti di dominio e di esclusione che le innervano. L'identità, compresa quella nazionale, è dunque un concetto assai più vasto e libero di quello che alcuni preferiscono cucirsi addosso, e, claustrofobicamente, declinare. Molteplice ed aperto, non indica una rigidità, ma un perenne fluire.

${ }^{19}$ N. Urbinati, Ai confini della democrazia. Opportunità e rischi dell'universalismo democratico, Roma, Donzelli, 2007, p.133.

${ }^{20}$ Non c'è nulla di ineluttabile nell'immigrazione, governarla si può, con intelligenza e realismo (come in tante altre cose, anche per l'immigrazione è questione di quantità, di proporzioni, di ritmi, di intensità, di regole e di equilibri), ma senza venir meno ai doveri di umanità e solidarietà internazionale. 
La questione, una volta di più, è quella - cruciale - dei processi di integrazione politica e sociale ${ }^{21}$. Cosa ci fa stare assieme? Come questo stare assieme può essere realizzato? Quali sono i processi che determinano il senso di appartenenza a una comunità politica? Nella modernità la nazione ha compensato il vuoto lasciato dalla religione con l'avvento della secolarizzazione (la democrazia, va ricordato, nasce e si sviluppa nel mondo moderno come democrazia "nazionale"). A svolgere quella funzione di unificazione politica sono intervenute nel Novecento i partiti e le identità politiche di massa e anche, in un certo senso, il Welfare State. Dopo il ridimensionamento della nazione (almeno in Europa) e la crisi delle appartenenze politico-ideologiche, cosa occupa oggi quel vuoto simbolico che caratterizza gli ordinamenti secolari22?

Come strumento di mobilitazione e unificazione politica c'è la tensione verso una società altra a cui allude la nostra Costituzione (quasi un recupero del nesso politico/simbolico: se ogni Costituzione democratica è un potente strumento di integrazione politica e di cambiamento sociale, non può fare a meno - citando Habermas - del senso di ciò che manca e perciò è ancora possibile ${ }^{23}$ ), ma si tratta di un'opzione svuotata sostanzialmente di significato e di prospettiva concreta nell'ambito dell'attuale architettura UE e dei vincoli imposti dalla globalizzazione neoliberale ${ }^{24}$.

${ }^{21}$ I processi di unificazione e integrazione politica sono generati dal concorso di più fattori: elementi formali (attinenti alla titolarità del potere), elementi materiali (attinenti agli interessi dei membri della comunità), elementi ideali (attinenti alla condivisione di valori). Cfr. M. LuCIANI, Costituzione, istituzioni e processi di costruzione dell'Unità Nazionale", in "Rivista AIC", n.2, 2011, p. 6.

22 Questo è uno degli interrogativi da cui muove l'analisi di G. Preterossi. Si veda G. Preterossi Ciò che resta della democrazia, Roma-Bari, Laterza, 2014, p. 24.

23 "La garanzia sul futuro" scrive G. Miglio "costituisce infatti il tratto di fondo dell'obbligazione politica; ci si obbliga politicamente soltanto in vista di una garanzia globale circa la soddisfazione dei bisogni che si immaginano incombere nel nostro avvenire. Un sistema politico conosce tanta più tensione verso l'unità interna [...] quanto più è tenuta desta l'attesa per il futuro; e il differimento nel tempo dei vantaggi e delle fruizioni si accompagna alla persuasione che soltanto in tal modo l'uomo possa realmente soddisfare i suoi bisogni futuri. Ma se non esiste obbligazione politica senza una immagine del tornaconto differito, senza un'ideologia utopica, il problema del tempo si rivela allora il tema fondamentale su cui la scienza è chiamata oggi a provarsi: un tema che si può affrontare soltanto sul terreno psicologico: appunto della psicologia del politico" (G. Miglio, Carl Schmitt, a cura di D. Palano, Brescia, Scholé - Editrice Morcelliana, 2018, p. 46). 


\section{Conclusioni}

Come ci ricorda il filosofo Kwame Anthony Appiah, mai come in quest'epoca "di sfide planetarie e interconnessione tra paesi c'è stato un bisogno più grande di un senso di destino umano condiviso" 25 . Spesso però chi fa professione di cosmopolitismo assomiglia a quel personaggio dei Fratelli Karamazov che scopre che quanto più ama

${ }^{24}$ A quali riserve di significato allora attingere per rigenerare il vincolo politico fra le persone? Sappiamo già che lo Stato contemporaneo, laico o secolarizzato, vive di presupposti che non può garantire. Ha bisogno cioè di riferimenti di valore metapolitici, senza $i$ quali il suo potere rischia di subire un processo di delegittimazione. Possiamo allora fare a meno qui in Italia di una "religione civile" capace di rinsaldare un rinnovato senso di appartenenza e di comunità? Per religione civile si intende qui - per citare G.E. Rusconi "l'insieme dei discorsi e degli atteggiamenti pubblici con valore e intento normativo, dotati di simbolismo e di codice religioso che si riferiscono alla formazione e affermazione della comunità nazionale. La nazione è un evento terrestre ma carico di senso trascendente. È un dato politico che pretende tuttavia un consenso metapolitico" (RUSCONI, Patria e Repubblica, Bologna, il Mulino, 1997, p. 20). Più in generale si tratta di riscoprire - per utilizzare termini di matrice neohegeliana - un terreno etico che sia comune e condiviso, di rilanciare cioè l'idea di un "ethos post-tradizionale e democratico" (A. HONNETH, Riconoscimento e disprezzo, Soveria Mannelli, Rubbettino, 1993, p.38) caratterizzato nel senso del pluralismo e dell' universalità. Tra l'altro, anche guardando alla politica, se questa non è più in grado "di produrre simboli si riduce alla semplice amministrazione tecnica dell'esistente; è una politica esangue, senza anima, destinata a soccombere" (G. DE LuNA, Una politica senza religione, Torino, Einaudi, 2013, p. 11), e questo vale soprattutto nelle fasi di maggior rottura della società, quando la tenuta dei legami sociali è più a rischio. Come a dire che la "religione civile" è una risorsa essenziale non soltanto per mantenere unita in ogni tempo una comunità politica, ma anche per far fronte alle sfide più difficili che il Paese incontra nella sua storia. E una democrazia sana non esiste senza una coscienza nazionale, che definisca ciò che i cittadini hanno in comune.

Lo stato secolarizzato, ad avviso di Böckenförde, non può non rifarsi a presupposti esterni, in quanto è incapace di produrre da sé le condizioni per la propria esistenza, cioè quegli incentivi alla cooperazione solidale che la secolarizzazione tende a erodere (si veda E.-W. BöCKENFÖRDE, Diritto e secolarizzazione. Dallo stato moderno all'Europa unita, Roma-Bari, Laterza, 2010). Pur muovendo da posizioni diverse, Jurgen Habermas riconosce oggi il rinnovato ruolo della religione quale fonte di motivazione che induce gli esseri umani alla cooperazione e alla responsabilità reciproca in vista della realizzazione secolare del regno di Dio. Proprio perché lo Stato liberale non può privarsi di importanti risorse di creazione del senso, Habermas sostiene che oggi esso si vede costretto non solo a permettere ma anche a favorire la partecipazione nel dibattito pubblico di credenti e rappresentanti delle comunità religiose in qualità di difensori di prospettive che, seppure non condivise dall'intera cerchia dei cittadini, contribuiscono alla diffusione di sentimenti di solidarietà.

${ }^{25}$ K. A. АртіAн "Mrs. May, We Are All Citizen of the World", Say Philosopher, in "BBC News", 29 ottobre 2016. 
l'umanità in generale, tanto meno considera le persone in particolare. I "cittadini del mondo" dovrebbero fare attenzione a che i lori ideali non diventino un pretesto per venir meno ai doveri che hanno nei confronti dei loro connazionali.

Scrive Dani Rodrik nel suo ultimo libro:

"Dobbiamo vivere nel mondo che abbiamo di fronte, con tutte le divisioni politiche, non in quello che desidereremmo avere. Il miglior modo per servire gli interessi globali è far fronte alle nostre responsabilità nel contesto delle istituzioni politiche che contano, ovvero quelle che esistono, all'interno dei confini nazionali" 26 .

Da una parte dobbiamo dunque accettare la complessità e la varietà del mondo e respingere qualsiasi ipotesi di reductio ad unum (che è inevitabilmente l'unità voluta dal più forte), perché lo spazio globale è irrimediabilmente plurale e articolato in entità geopolitiche organizzate per grandi spazi con le grandi potenze a contendersi di volta in volta un ruolo egemonico mondiale (così come le comunità nazionali si organizzano attorno ad una dialettica interno/esterno, lo stesso avviene quando sono più paesi a unirsi fra loro secondo linee e sfere di influenza); dall'altra non bisogna rinunciare alla costruzione di momenti di unità e condivisione di responsabilità fra gli Stati, al fine di pervenire a forme di stabilità in grado di assicurare la pace e la giustizia fra le nazioni. Parafrasando Gramsci, se il "punto di partenza è nazionale", la vocazione non può che essere internazionalista: questo significa farsi costantemente carico dei problemi del mondo, rilanciare un senso aperto di una comune appartenenza, mobilitarsi contro chiunque offenda il principio di autodeterminazione dei popoli e la dignità della persona umana (vedi il dramma infinito vissuto dai palestinesi nel silenzio colpevole della comunità internazionale), credere infine nella capacità degli essere umani di costruire un mondo migliore.

${ }^{26}$ D. RoDrIK, Dirla tutta sul mercato globale, Torino, Einaudi, 2018, p. 47. 
Abstract - Watching the history of mankind, epidemics represent a tragic constant; a few of them - like Coronavirus - involve the whole planet. It was in the years of the Naples cholera when Leopardi wrote $L a$ ginestra, his masterpiece of poetry and phi- losophy. Nowadays, as we face the effects of a new pandemic, it may be useful to retrace Leopardi's route, that right with La ginestra "jumps" from patriotism to universal brotherhood, without establishing or assuming any contradiction between the two moments. 